International Journal of Biomedical Engineering and Science (IJBES), Vol. 3, No. 3, July 2016

\title{
POWER SPECTRAL ANALYSIS OF EEG AS A POTENTIAL MARKER IN THE DIAGNOSIS OF SPASTIC CEREBRAL PALSY CASES
}

\author{
Bablu Lal Rajak ${ }^{1}$, Meena Gupta ${ }^{1}$, Dinesh Bhatia ${ }^{* 1}$, Arun Mukherjee ${ }^{2}$, \\ Sudip Paul ${ }^{1}$, Tapas Kumar Sinha ${ }^{3}$ \\ ${ }^{1}$ Department of Biomedical Engineering, North Eastern Hill University, \\ Shillong-793022, Meghalaya \\ ${ }^{2}$ UDAAN-for the differently abled, Lajpat Nagar, New Delhi-110024 \\ ${ }^{3}$ Computer Centre, North Eastern Hill University, Shillong-793022, Meghalaya \\ *Corresponding author: Dr. Dinesh Bhatia, Department of Biomedical Engineering, \\ North Eastern Hill University, Shillong-793022, Meghalaya.
}

\begin{abstract}
The detection and diagnosis of various neurological disorders are performed using different medical devices among which electroencephalogram (EEG) is one of the most cost effective technique. Though significant progress had been made in the analysis of EEG for diagnosis of different neurological disorders, yet detection of cerebral palsy $(C P)$ is not quite clear. This study was performed to analyze the EEG power spectrum density (PSD) of spastic CP and normal children to find if any significant EEG patterns could be used for early detection of CP. Twenty children participated in this study out of which ten were spastic $C P$ and other ten were normal healthy children. EEG of all the participants was recorded from C3 C4 and F3 F4 regions following montage 10-20 system. The artifact-free EEG signals of 15 minutes duration was extracted for spectral analysis using Fast Fourier Transformation (FFT) algorithm in MATLAB and power density spectrum (PSD) was plotted. The PSD revealed high intensity power peak at frequency of $50 \mathrm{~Hz}$ and smaller at $100 \mathrm{~Hz}$, which was consistent for all healthy subjects. In case of spastic CP children, high intensity peak at $100 \mathrm{~Hz}$ were prominent and smaller peak was observed at $50 \mathrm{~Hz}$. The high intensity $100 \mathrm{~Hz}$ peak observed in the PSD of spastic CP patients demonstrated that this tool can be used for early detection of spastic CP.
\end{abstract}

\section{KEYWORDS:}

Cerebral palsy, electroencephalogram (EEG), Fast Fourier Transformation (FFT), power spectrum density $(P S D)$

\section{INTRODUCTION}

Brain's electrical activity had been continuously studied to understand its function or to diagnose neurological disorders. Various medical imaging devices are employed for the diagnosis of neurological disorders; but none of these are as cost effective and practical as using an electroencephalogram (EEG). EEG recordings are obtained by placing the electrodes on the surface of the scalp to acquire signals that have a frequency range which spans from 0 to $100 \mathrm{~Hz}$ where different frequency bands represent different activity levels [1]. EEG provides real time DOI: $10.5121 /$ ijbes.2016.3303 
pictorial information of the cerebral activity that help in the identification and classification of a number of neurological disorders in both pediatric and adult population [2]. Recently, EEG is widely used to detect brain's neurological dysfunction such as epilepsy, Parkinson's disease, Alzheimer's disease, etc. [3,4,5]; and in the study of mental stress [6], effect of anesthesia [7], dyslexia[8], and so on; but the role of EEG in detection of cerebral palsy (CP) has not been well established $[9,10]$.

Cerebral palsy also known as static encephalopathy causes abnormality of movement and posture; it occurs due to lesion of a developing brain in children below the age of two years. CP results in different movement patterns including spastic, dyskinetic, hypotonic, ataxic, and mixed forms; among which the most common is spastic affecting almost 70 to $80 \%$ of the patients [11]. The motor disorder in CP is often accompanied by disturbances of sensation, perception, cognition, communication, behavior, epilepsy and secondary musculoskeletal problems [12]. These problems arise due to abnormal brain activity in these children which was noted by Perlsteinet al (1955), who studied $1217 \mathrm{CP}$ patients and found that $90 \%$ of them had abnormal electroencephalogram (EEG) recordings [13]. Similar conclusions were given by Lindsley and Jones (1956); Melin (1962) and Al-Sulaiman (2001) after performing EEG studies on CP patients $[14,15,16]$. Thus this study was aimed at analyzing the EEG power spectrum density (PSD) of spastic CP and normal children to find if any significant EEG patterns were presentwhich could be used for early detection of CP.

Power spectral analysis is one of the widely used methods for quantification of EEG signal. The PSD provides 'frequency content' of the signal or the distribution of power over frequency. PSD analysis is a mathematical method for frequency analysis of complex waveforms, which provides a sensitive means for detecting periodicity within the waveforms and determining the relative energy content of the periodicities. This method has been useful in computerized analysis of EEG using Fourier transformations [17].

\section{MATERIALS AND METHODS}

\subsection{Participants}

Twenty children were recruited in this study after informed consent from their parents or guardians and approval by the Institutional Ethics Committee on Human Samples or Participants. Ten participants were spastic CP children (7 male and 3 female, mean age: $8.1 \pm$ SD 4.20) and other ten were normal healthy children (6 male and 4 female, mean age: $8.6 \pm$ SD 3.31) not suffering from any neurological disorders. The spastic CP children were screened on the basis of their reports from registered medical practitioner and physiotherapists. The recruited children (both CP and normal) were from the out-patient department of UDAAN-for the differently abled, a non-profit organization in Delhi that has pioneered in rehabilitation based treatment using physical, occupational, speech therapy since 1992 and it also runs anoutreach learning program for the normal children of nearby areas from the low income group families.

\subsection{EEG recording}

The EEG recording on the participants was performed using Nexus Mark II, neurofeedback system (Mind Media B.V., Netherlands). Prior to recording, the participants underwent a preparatory procedure, where the participants were asked to sit on the chair and relax. The target 
recording areas on their scalp was cleaned using alcohol swabs and the metal electrodes were fixed into those areas using a conductive gel. The selected recording areas were F3, F4 and C3, $\mathrm{C} 4$; the reference electrode was placed at right mastoid (figure 1).The electrodes were placed according to the internationally recognized Montage 10-20 system [18]. After the electrode placement was completed, the recording was started which continued for 20 minutes at an output rate of 256 samples per second using a 2 channel bipolar electrodes. The entire recording of EEG signal was performed without using any anesthetics and hence the participants were awake during the process. After completion of the recording, electrodes were removed and the conductive gel on the scalp of the patients was washed with water.
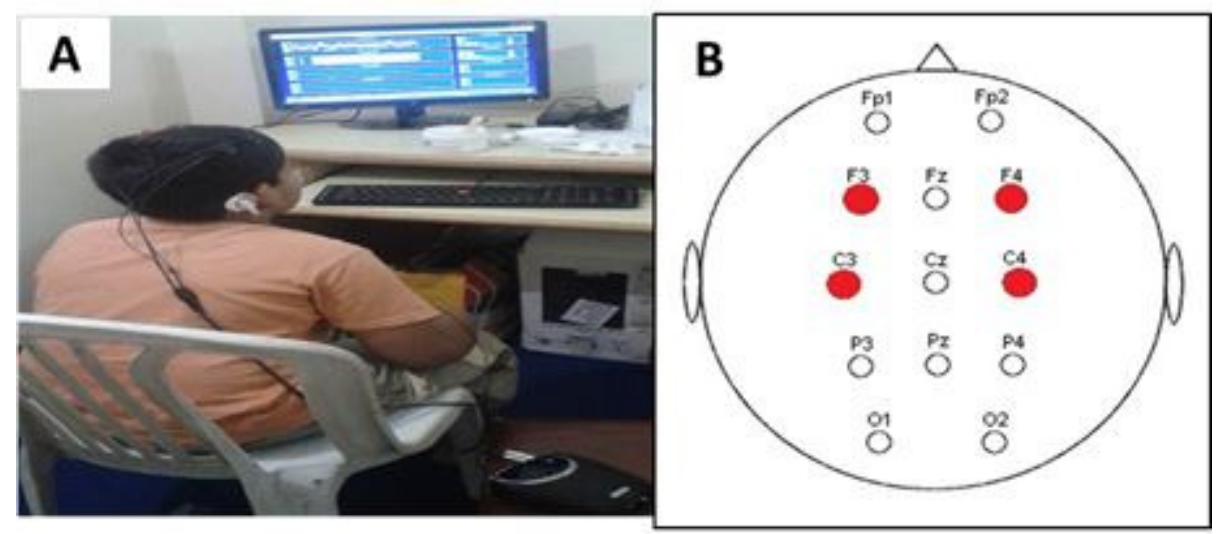

Figure1: A) EEG recording of a normal child: B) 10-20 showing recording areas

\subsection{Data Analysis}

The recorded EEG signal was imported in an ANSI text file format from the Biotrace+ software that runs the Nexus Mark II, neuro feedback system. Pre-processing of EEG signals such as removal of artifact and noise was performed using the software prior to converting the signals into ANSI text file.The ANSI text file was used for data analysis in MATLAB. The EEG data of 15 minutes was used for analysis with Fast Fourier transformation using Welch method to obtain the PSD which were plotted power versus frequency. Welch is a non-parametric method achieved by dividing the signal sequence into segments, multiplying the segment with an appropriate window and calculation of the periodogram by computing the squared magnitude on the result of its discrete Fourier transform. Individual periodogram obtained are then averaged, resulting in the measurement of power in relation to frequency [19]. Welch method offers to reduce noise if compared to the standard periodogram with fewer computations.

In this method the data sequences $x_{i}(n)$ can be represented as

$$
x_{i}(n)=x(n+i D) \quad \text { here } n=0,1,2, \ldots, M-1 \text { and } I=0,1,2, \ldots, L-1
$$

where $i D$ is the beginning of $i$ th sequence. This will lead to formation of $L$ data segments each of length $2 M$. The modified periodogram is given as: 
International Journal of Biomedical Engineering and Science (IJBES), Vol. 3, No. 3, July 2016

$$
\tilde{P}_{x x}^{(i)}(f)=\left|\frac{1}{M U} \sum_{n=0}^{M-1} x(n) \omega(n) e^{-j 2 \pi f n}\right|^{2}
$$

where $U$ is the normalization factor for the power in the window function given as: $U=\frac{1}{M} \sum_{n=0}^{M-1} \omega^{2}(n)$ and $\omega(n)$ is the window function. Finally the Welch power spectrum which is the average of these periodogram is given as:

$$
P_{x x}^{W}(f)=\frac{1}{L} \sum_{i=0}^{L-1} \tilde{P}_{x x}^{(i)}(f)
$$

\section{RESULTS}

From the PSD of EEG signal, it was observed that there were two power peaks at frequency $50 \mathrm{~Hz}$ and $100 \mathrm{~Hz}$. These power peaks were common, both in spastic CP and normal children; though slight variation such as power intensity and slight frequency shift (between $100 \mathrm{~Hz}$ and $103 \mathrm{~Hz}$ ) was observed in different participants. The results from different areas of the brain and from different participant type are discussed below:

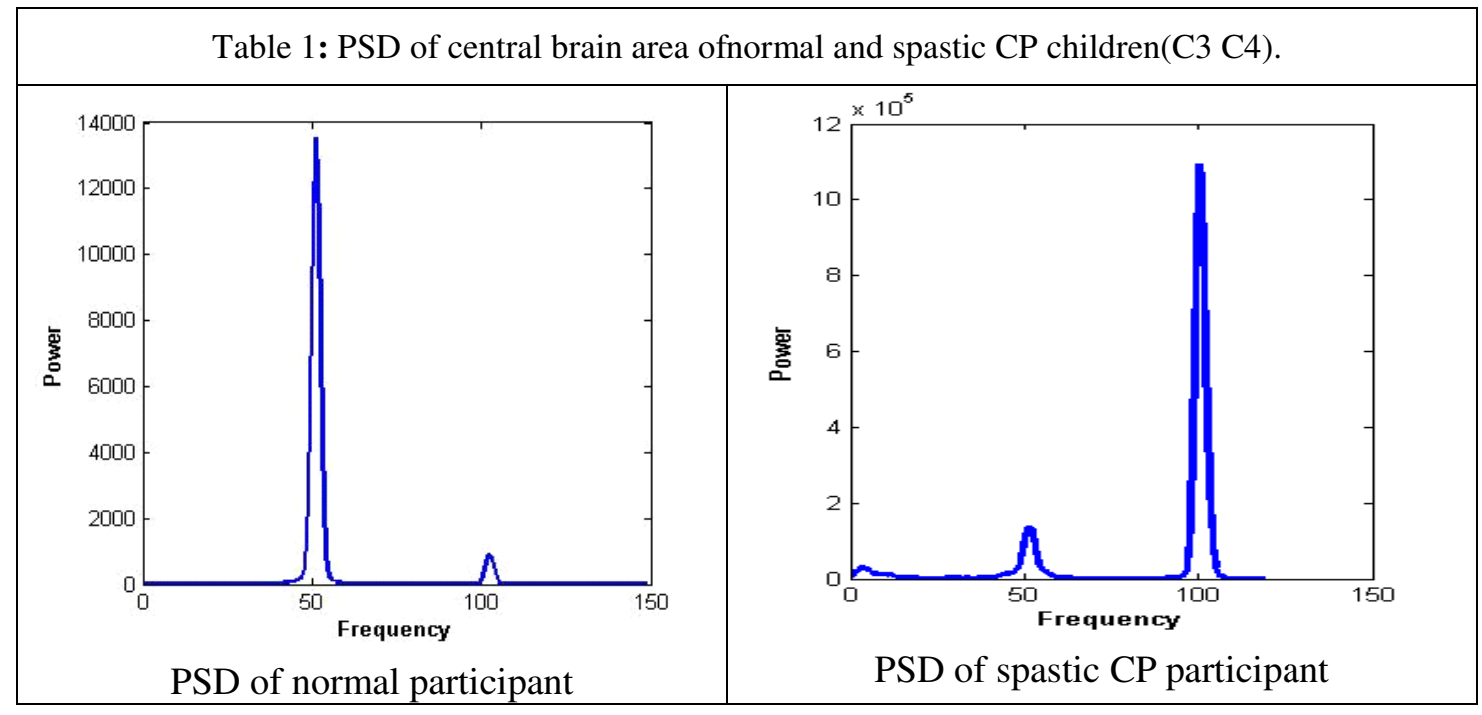

\section{Case 1:}

Table 1 represents two PSD plots of normal and spastic CP participants from the central area of the brain (C3and C4). The power peak of high intensity was consistently observed in all the normal childrenat frequency $50 \mathrm{~Hz}$ and a small peak at $100 \mathrm{~Hz}$. In case of, spastic CP children, the power peak of higher intensity was observed at frequency $100 \mathrm{~Hz}$ in 9 of the participants and comparatively low intensity peak at $50 \mathrm{~Hz}$ were present 


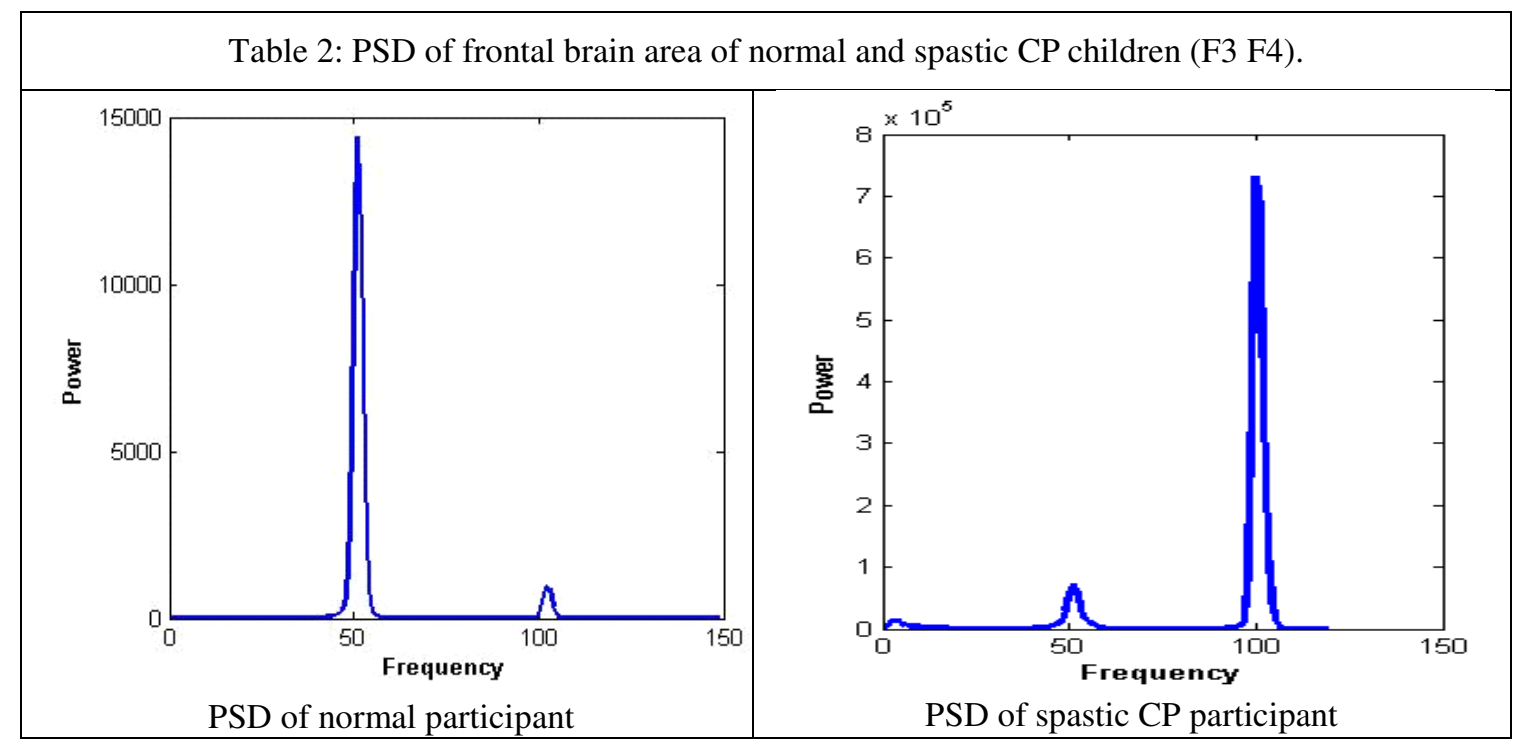

\section{Case 2:}

Similar to the PSD plots of central region, PSD of frontal area of normal and spastic CP participants also showed two peaks at $50 \mathrm{~Hz}$ and $100 \mathrm{~Hz}$. Two sample PSD plots of normal and spastic CP participants from the frontal area of the brain (F3 andF4)are shown in table 2. The power peak of high intensity was consistently found in all the normal children at frequency $50 \mathrm{~Hz}$, along with a small peak at $100 \mathrm{~Hz}$. In spastic CP children, the power peak of higher intensity was observed at frequency $100 \mathrm{~Hz}$ in eight of the participants along with a low intensity peak at $50 \mathrm{~Hz}$.

The high intensity peaks at frequency $100 \mathrm{~Hz}$ observed in spastic CP patients could be due to the abnormal electrophysiological brain activity that affects their movement and posture, since the EEG recording areas used in this study are known for movement and motor planning.

\section{DISCUSSION}

Though significant progress had been made in the analysis of EEG signals using various algorithms yet the diagnosis of $\mathrm{CP}$ are still based on clinical assessment and neuroimaging findings such as Magnetic Resonance Imaging, Computer Tomography, Cranial Ultrasound and so on [20]. However, in the presence of associated seizures, it was essential for the electrical characterization of the seizure discharge, which is performed using EEG. As reported by previous researchers [15-18], EEG of CP patients demonstrate abnormal activity, thus we decided to find some pattern in the acquired signal using a FFT based algorithm such that EEG of these patients 
International Journal of Biomedical Engineering and Science (IJBES), Vol. 3, No. 3, July 2016

can be used for diagnostic purpose. If proven and established, this technique could be beneficial in examining large number of cases at comparatively lower costs. In our study, we used FFT based program in MATLAB to perform power spectral analysis of spastic CP and normal children. It was observed that PSD of spastic CP children from both central and frontal areas of the brain showed high intensity peaks at $100 \mathrm{~Hz}$ which could be due to high neuronal activity in the motor areas that is responsible for their uncoordinated movement and posture. The $100 \mathrm{~Hz}$ peak was observed in nine (9) out of the 10 children in the central region and eight (8) out of 10 in the frontal region of spastic CP children. PSD of 1 child in central and 2 children in frontal region showed a number of peaks at $3 \mathrm{~Hz}, 20 \mathrm{~Hz}, 51 \mathrm{~Hz}$ and $103 \mathrm{~Hz}$ which could be due to errors during signal acquisition. Nevertheless, these findings are significant steps towards development of diagnostic tool for the detections of CP using cost effective EEG signals.

However, our study had some limitations - first, the study had a small sample size since we were considering only spastic CP patients due to their availability in the rehabilitation center. Second, the EEG signals were taken from $\mathrm{C} 3 \mathrm{C} 4$ and $\mathrm{F} 3 \mathrm{~F} 4$ position only and not from any other parts of the brain. Third, PSD was obtained using only FFT- Welch method and no other feature extraction algorithm were employed.

\section{CONCLUSION AND FUTURE WORKS}

Previous researches had shown that $\mathrm{CP}$ patients have abnormal EEG but still the diagnosis of this disorder was not performed using EEG even thoughrecording of this signal is comparatively simple and cost effective. In this study we used the EEG signals of both normal and spastic CP participants to extract some meaningful feature using FFT-Welch method. The result of our study demonstrate that FFT based algorithm can be used to convert raw EEG signals into meaningful information that can be used for diagnosis purpose. The high intensity $100 \mathrm{~Hz}$ peak in the PSD of spastic CP patient was consistent in the central and frontal areas of the brain; demonstrating that this can be used as potential markers in the early diagnosis of CP.Furthermore, in our future work we will consider more parameters which can demonstrate early diagnosis of different types of cerebral palsy.

\section{ACKNOWLEDGEMENT}

This work is supported by funding received (Ref: SEED/TIDE/007/2013) from the Technology Intervention for Disabled and Elderly (TIDE) of the Department of Science and Technology (DST), Government of India, New Delhi to North Eastern Hill University, Shillong, Meghalaya, 793022, India. The authors also acknowledge the support of all the therapists and staff of UDAAN-for the disabled, Delhi. A special thanks to all the participating children and their parents. 
International Journal of Biomedical Engineering and Science (IJBES), Vol. 3, No. 3, July 2016

\section{REFERENCES}

1. E. Niedermeyer, "Epileptic seizure disorders" Chapt. 27, in E. Niedermeyer and F.L. da Silva ed. Electroencephalography: Basic principles, Clinical applications, and Related fields", $4^{\text {th }}$ ed. Lippincott Willams\& Wilkins, Philadelphia, 1999.

2. H. Adeli and S. Ghosh-Dastidar,"Automated EEG-based diagnosis of neurological disorders: Inventing the future of neurology", CRC press, 2010.

3. Smith SJ.EEG in the diagnosis, classification, and management of patients with epilepsy.Journal of Neurology, Neurosurgery \& Psychiatry. 2005 Jun 1;76(suppl 2):ii2-7.

4. J. Dauwels, F. Vialatte, T. Musha, A. Cichocki,“A comparative study of synchrony measures for the early diagnosis of Alzheimer's disease based on EEG", NeuroImage, vol. 49, no. 1, p. 668-93, Jan 2010.

5. R. Wang, J. Wang, H. Li, Y. Chen, "Power spectral density and high order bispectral analysis of Alzheimer's EEG", InControl and Decision Conference (CCDC), 2015 IEEE 27th Chinese, p. 1822-1826, May 2015.

6. W. E. Kincses, S. Hahn, P. D. Schrauf, E. A. Schmidt,"Measuring driver's mental workload using EEG", ATZ Worldwide, vol. 110, no. 3, p.12-7, Mar 2008.

7. O. Dressler, G. Schneider, G. Stockmanns, E.F. Kochs, "Awareness and the EEG power spectrum: analysis offrequencies", British Journal of Anesthesia, vol. 93, no. 6, p. 806-9, Dec 2004.

8. Z. Mahmoodin, W. Mansor, K. Y. Lee, N. B. Mohamad,"An analysis of EEG signal power spectrum density generated during writing in children with dyslexia", InSignal Processing \& Its Applications (CSPA), 2015 IEEE 11th International Colloquium, p.156-160, Mar 2015.

9. J. Foley, "Deterioration in the EEG in children with cerebral palsy", Developmental Medicine \&Child Neurology, vol. 10, no. 3, p. 287-301, Jun 1968.

10. N. Gordon, "The electroencephalogram in cerebral palsy", Developmental Medicine \&Child Neurology, vol. 8, no. 2, p. 216-218, Apr 1966.

11. M. Yeargin-Allsopp, K. V. N. Braun, N. S. Doernberg, R. E. Benedict, R. S. Kirby, M. S. Durkin, "Prevalence of cerebral palsy in 8-year-old children in three areas of the United States in 2002: a multisite collaboration", Pediatrics, vol. 121, no. 3, p. 547-554, Mar 2008.

12. M. Bax, M. Goldstein, P. Rosenbaum, A. Leviton, N. Paneth, B. Dan, et al.,"Proposed definition and classification of cerebral palsy, April 2005", Developmental Medicine\&Child Neurology, vol. 47, no. 8, p. 571-576, Aug 2005.

13. M. A. Perlstein, H. Gibbs, F. A. Gibbs, "The electroencephalogram in infantile cerebral palsy", American Journal of Physical Medicine \& Rehabilitation, vol. 34, no. 4. P. 477-96, Aug 1955.

14. D. Lindsley and M. Jones, "Clinical and EEGcorrelations in cerebral palsy children and adults", Electroencephalogrraphy\&ClinicalNeurophysiology, vol. 8, no. 1, p. 168, Jan 1956.

15. K. A. Melin,"EEG and epilepsy in cerebral palsy", Developmental Medicine\&Child Neurology, vol. 4, no. 2, p. 180-3, Apr 1962.

16. A. Al-Sulaiman, "Electroencephalographic findings in children with cerebral palsy: a study of 151 patients", Functional neurology, vol. 16, no. 4, p. 325-8, Oct 2001.

17. G. Dumermuth and R. Fluhler, "Some modern aspects in numerical spectrum analysis of multichannel electroencephalographic data", Med.Biol.Engin., vol. 5, no. 4, p. 319-331, Jul 1967.

18. H. H. Jasper, "The ten twenty electrode system of the international federation", Electroencephalography and clinical neurophysiology, vol. 10, p. 371-5, 1958.

19. P. D. Welch, "The use of fast Fourier transform for the estimation of power spectra: A method based on time averaging over short, modified periodograms", IEEE Transactions on audio and electroacoustics, vol. 15, no. 2, p. 70-3, 1967.

20. M. Bosanquet, L. Copeland, R. Ware, R. Boyd, "A systematic review of tests to predict cerebral palsy in young children", Developmental Medicine \& Child Neurology, vol. 55, no. 5, p. 418-26, May 2013. 